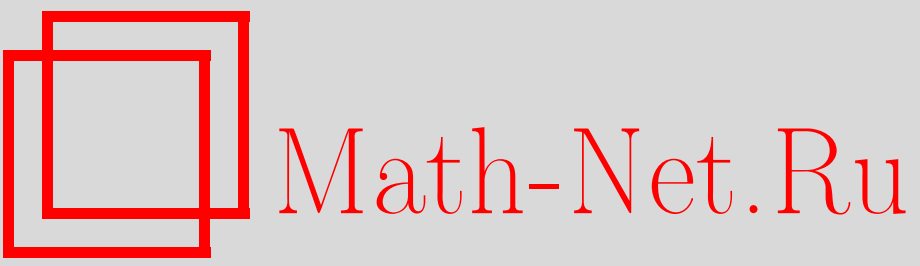

С. А. Игонин, Горизонтальные когомологии с коэффициентами и нелинейные представления нулевой кривизны, УМН, 2003, том 58, выпуск 1, 185-186

DOI: https://doi.org/10.4213/rm600

Использование Общероссийского математического портала Math-Net.Ru подразумевает, что вы прочитали и согласны с пользовательским соглашением

http://www.mathnet.ru/rus/agreement

Параметры загрузки:

IP: 54.89 .56 .158

26 апреля 2023 г., 09:09:23 


\title{
ГОРИЗОНТАЛЬНЫЕ КОГОМОЛОГИИ С КОЭФФИЦИЕНТАМИ И НЕЛИНЕЙНЫЕ ПРЕДСТАВЛЕНИЯ НУЛЕВОЙ КРИВИЗНЫ
}

\author{
С.А. Игонин
}

Рассмотрим инволютивную систему дифференциальных уравнений

$$
F_{s}\left(x_{i}, u^{q}, u_{x_{i_{1}} \ldots x_{i_{k}}}^{q}, \ldots\right)=0, \quad i=1, \ldots, N, \quad q=1, \ldots, M, \quad s=1, \ldots, L,
$$

задающую со своими дифференциальными следствиями подмногообразие $\mathscr{E}$ в соответствующем пространстве бесконечных джетов [1]-[3]. Практически все уравнения математической физики таковы. В теории солитонов [4] переопределенные системы вида

$$
v_{x_{i}}^{j}=A_{i}^{j}\left(v^{k}, x_{r}, u^{q}, u_{x_{i_{1}} \ldots x_{i_{k}}}^{q}, \ldots\right), \quad j=1, \ldots, m, \quad i=1, \ldots, N,
$$

совместные в силу (1), играют важную роль. Если функции $A_{i}^{j}$ линейны по $v^{k}$, то (2) задает линейное представление нулевой кривизны (ЛПНК) системы (1) в алгебре Ли $\mathfrak{g l}_{m}(\mathbb{R})$ [4], [5]. Нелинейные системы (2) часто порождают преобразования Бэклунда для (1) [4], [6].

В работах [5], [7] были введены гомологии, помогающие описать все ЛПНК в матричных алгебрах Ли для данной системы (1). Усилив резултаты [3], мы определяем аналогичные гомологии для произвольных систем (2) и используем их для изучения структуры (2).

Для гладкого многообразия $M$ обозначим через $D^{p}(M)$ модуль дифференцирований алгебры $C^{\infty}(M)$ со значениями в модуле $p$-форм и через $[*, *]: D^{p}(M) \times D^{q}(M) \rightarrow D^{p+q}(M)$ скобку Нийенхейса [1]. Тогда $D^{0}(M)=D(M)$ совпадает с алгеброй Ли векторных полей.

Геометрическая интерпретация (2) такова [1], [6]. Полные производные $D_{x_{i}} \in D(\mathscr{E})$ порождают распределение Картана $\mathscr{C} \subset D(\mathscr{E})$. Структура накрытия в тривиалњном расслоении $\pi: \mathscr{E} \times W \rightarrow \mathscr{E}, \operatorname{dim} W<\infty$, задается таким распределением $\mathscr{D} \subset D(\mathscr{E} \times W)$, что $[\mathscr{D}, \mathscr{D}] \subset \mathscr{D}$ и $\left.\pi_{*}\right|_{\mathscr{D}}-$ изоморфизм на $\mathscr{C}$. Два накрытия $\mathscr{D}_{1}, \mathscr{D}_{2} \subset D(\mathscr{E} \times W)$, связанные соотношением $\mathscr{D}_{1}=G_{*} \mathscr{D}_{2}$, где $G: \mathscr{E} \times W \rightarrow \mathscr{E} \times W$ - послойньй диффеоморфизм, назьваются эквивалентными. Накрытия, эквивалентные $\mathscr{D}=\nabla(\mathscr{C})$, где $\nabla$ - тривиальная связность в $\pi$, называются тривиальными. Для $X \in \mathscr{C}$ обозначим через $\widetilde{X}$ такое векторное поле из $\mathscr{D}$, что $\pi_{*}(\widetilde{X})=X$.

Распределение $\mathscr{D}$ порождено полями $\widetilde{D} x_{i}$. Система (2) определяет накрытие $\pi: \mathscr{E} \times \mathbb{R}^{m} \rightarrow \mathscr{E}$, $\widetilde{D}_{x_{i}}=D_{x_{i}}+\sum_{j} A_{i}^{j} \partial_{v^{j}}$, и всякое накрытие локально имеет такой вид. Эквивалентные накрытия получаются заменой переменных $\widetilde{x}_{i}=x_{i}, \widetilde{u}^{q}=u^{q}, \widetilde{v}^{j}=f^{j}\left(v^{k}, x_{r}, u^{q}, \ldots\right)$ в (2). Накрытие (2) тривиальн, если такой заменой его можно привести к виду $\widetilde{v}_{x_{i}}^{j}=0$.

Положим $\mathscr{F}=C^{\infty}(\mathscr{E}), \widetilde{\mathscr{F}}=C^{\infty}(\mathscr{E} \times W)$. Рассмотрим подмодуль $D_{v}^{p, q} \subset D^{p+q}(\mathscr{E} \times W)$ $\pi$-вертикальных дифференцирований со значениями в $\widetilde{\mathscr{F}}$-подмодуле, порожденном формами $\pi^{*}\left(\mathscr{C}^{p} \Lambda \otimes_{F} \Lambda_{h}^{q}\right)$, где $\mathscr{C}^{p} \Lambda$ и $\Lambda_{h}^{q}-$ модули картановских и горизонтальных форм на $\mathscr{E}[1]$. Элемент $\widetilde{d}_{h}=\sum_{i} d x_{i} \widetilde{D}_{x_{i}} \in D^{1}(\mathscr{E} \times W)$ удовлетворяет $\left[\widetilde{d}_{h}, \widetilde{d}_{h}\right]=0$ и задает комплекс

$$
D_{v}^{p, 0} \stackrel{\partial_{\mathscr{D}}}{\longrightarrow} D_{v}^{p, 1} \stackrel{\partial_{\mathscr{D}}}{\longrightarrow} D_{v}^{p, 2} \stackrel{\partial_{\mathscr{D}}}{\longrightarrow} \cdots, \quad \partial_{\mathscr{D}}(w)=\left[\widetilde{d}_{h}, w\right] .
$$

Будем рассматривать пространство $D_{v} \subset D(\mathscr{E} \times W) \pi$-вертикальных векторных полей как (бесконечномерный) модуль над $\mathscr{F} \subset \widetilde{\mathscr{F}}$. Тогда это $\mathscr{C}$-модуль [1], [3] с действием $X(Y)=[\tilde{X}, Y]$, $X \in \mathscr{C}, Y \in D_{v}$, алгебры Ли $\mathscr{C}$. Имеем $D_{v}^{p, q} \cong D_{v} \otimes_{\mathscr{F}} \mathscr{C}^{p} \Lambda \otimes_{\mathscr{F}} \Lambda_{h}^{q}$ и комплекс (3) изоморфен горизонталшному комплексу де Рама на $\mathscr{E}$ с коэффициентами в $D_{v} \otimes \mathscr{C}^{p} \Lambda$.

Горизонтальные когомологии с коэффициентами в проективном $\mathscr{C}$-модуле $Q$ изучались в [1], [3]. Однако проективность там используется только чтобы показать, что точность строк некоторого бикомплекса сохраняется после тензорного умножения на $Q$. Но эти строки являются точными последовательностями векторных расслоений над $\mathscr{E}$, поэтому они расщепляются и остаются точными после умножения на любой модуль $Q$. Справедлива

Теорема 1. Когомологические изоморфизмы из [3] верны для любых $\mathscr{C}$-модулей $Q$. $B$ частности, $i$-я группа когомологий горизонтального комплекса де Рама на Е с коәффициентами в $Q \otimes \mathscr{C}^{1} \Lambda$ изоморфна $(N-i)$-й группе гомологий комплекса $\widehat{P}_{*} \otimes Q$, где 
$\widehat{P}_{*}$ - сопряженный комплекс для комплекса совместности (комплекса Жане) системы (1) [1]-[3].

Длина $k_{\mathscr{E}}$ комплекса совместности отражает степень переопределенности системы $(1)$, для непереопределенных невырожденных систем имеем $k_{\mathscr{E}}=2$. Рассмотрим поднятия $d_{h}, d_{\mathscr{C}} \in$ $D^{1}(\mathscr{E} \times W)$ с помощью $\nabla$ горизонтального и картановского дифференциалов с $\mathscr{E}$. Из $\left[\widetilde{d}_{h}, \widetilde{d}_{h}\right]=$ $\left[d_{\mathscr{C}}, d_{h}\right]=0$ имеем $\partial_{\mathscr{D}}\left(\left[d_{\mathscr{C}}, \widetilde{d}_{h}-d_{h}\right]\right)=0$. Аналогично [5; раздел 4$]$ верна

ТЕОРема 2. Пусть $k_{\mathscr{E}} \leqslant N$. Структура накрытия $\mathscr{D}$ в конечномерном расслоении $\mathscr{E} \times W \rightarrow \mathscr{E}$ локально тривиальна тогда и только тогда, когда образ $\chi_{\mathscr{D}} \in H_{N-1}\left(\widehat{P}_{*} \otimes\right.$ $\left.D_{v}\right)$ класса $\left[d_{\mathscr{C}}, \widetilde{d}_{h}-d_{h}\right] \in H^{1}\left(D_{v}^{1, *}\right)$ при изоморфизме теоремь 1 равен нулю.

Если $k_{\mathscr{E}}<N$, то $H_{N-1}\left(\widehat{P}_{*} \otimes D_{v}\right)=0$, поэтому получаем

СлеДСтвиЕ. При $k_{\mathscr{E}}<N$ все накрытия над $\mathscr{E}$ локально тривиальны. В частности, для непереопределенных уравнений (1) при $N>2$ все системы (2) тривиальны.

Выпишем формулы для $\chi \mathscr{D}$ при $N=k_{\mathscr{E}}=2$, обобщающие формулы [7; раздел 3] для ЛПНК. Обозначим $A_{i}=\sum_{j} A_{i}^{j} \partial_{v}$. Совместность (2) в силу (1) равносильна тождеству в пространстве джетов $\left[D_{x_{1}}+A_{1}, D_{x_{2}}+A_{2}\right]=\sum_{p, q, s} D_{x_{1}}^{p} D_{x_{2}}^{q}\left(F_{s}\right) \cdot g_{p q}^{s}$ для некоторых векторных полей $g_{p q}^{s}=\sum_{j} g_{p q}^{s j}\left(v^{k}, x_{i}, u^{r}, \ldots\right) \partial_{v j}$. Класс $\chi_{\mathscr{D}}$ задается набором векторных полей из $D_{v}$ :

$$
\chi_{\mathscr{D}}^{s}=\sum_{p, q}(-1)^{p+q} \widehat{D}_{x_{1}}^{p} \widehat{D}_{x_{2}}^{q}\left(\left.g_{p q}^{s}\right|_{\mathscr{E} \times W}\right), s=1, \ldots, L, \widehat{D}_{x_{i}}(g)=D_{x_{i}}(g)+\left[A_{i}, g\right] \forall g \in D_{v} .
$$

Теорема 3. Пусть $N=k_{\mathscr{E}}=2$. При переходе $\kappa$ әквивалентному накрытию $\mathscr{D}^{\prime}=$

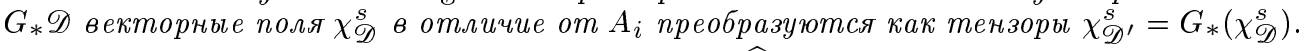
Условие, что $\chi_{\mathscr{D}}$ является 1-циклом комплекса $\widehat{P}_{*} \otimes D_{v}$, имеет вид

$$
\sum_{s, p, q}(-1)^{p+q} \widehat{D}_{x_{1}}^{p} \widehat{D}_{x_{2}}^{q}\left(\frac{\partial F_{s}}{\partial u_{p, q}^{r}} \chi_{\mathscr{D}}^{s}\right)=0, \quad r=1, \ldots, M, \quad \text { əде } \quad u_{p, q}^{r}=\frac{\partial^{p+q} u^{r}}{\partial x_{1}^{p} \partial x_{2}^{q}} .
$$

Уравнения (4) в дополнениек $\left.\left[D_{x_{1}}+A_{1}, D_{x_{2}}+A_{2}\right]\right|_{\mathscr{E}}=0$ помогают описывать накрытия. Например, для накрытия $v_{x}^{j}=A_{1}^{j}, v_{t}^{j}=A_{2}^{j}$ над эволюционной системой $u_{t}^{s}=f_{s}\left(x, t, u^{a}, u_{n}^{a}, \ldots\right)$, где $u_{n}^{a}=\partial u^{a} / \partial x^{n}$, имеем $\chi_{\mathscr{D}}^{s}=\sum_{n}(-1)^{n} \widehat{D}_{x}^{n}\left(\partial_{u_{n}^{s}} A_{1}\right)$. Из условия (4) следует

Теорема 4. Для әволюционного уравнения $u_{t}=f\left(x, t, u, u_{n}, \ldots\right)$ четного порядка существует такое $O \in \mathbb{N}$, что любое накрытие эквивалентно накрытию с $\partial A_{i}^{j} / \partial u_{n}=0$ $\forall n \geqslant O$. Для нелокального закона сохранения $\widetilde{D}_{t}(\varphi)=\widetilde{D}_{x}(\psi)$ этого уравнения существует әквивалентный $\varphi^{\prime}=\varphi+\widetilde{D}_{x}(\rho), \psi^{\prime}=\psi+\widetilde{D}_{t}(\rho)$ с $\partial \varphi^{\prime} / \partial u_{n}=\partial \psi^{\prime} / \partial u_{n}=0 \quad \forall n \geqslant O$.

Для локальных законов сохранения $D_{t}(\varphi)=D_{x}(\psi)$ это утверждение хорошо известно. Автор благодарит А. М. Вербовецкого и И. С. Красилшцика за полезные обсуждения.

\section{СПИСОК ЛИТЕРАТУРЫ}

[1] J. Krasilshchik, A. Verbovetsky // e-print math.DG/9808130. [2] T. Tsujishita // Differential Geom. Appl. 1991. V. 1. № 1. P. 3-34. [3] A. Verbovetsky // Contemp. Math. 1998. V. 219. P. 211-231; e-print math.DG/9803115. [4] А. Ньюэлл. Солитоны в математике и физике. М.: Мир, 1989. [5] M. Marvan // Proceedings of the Fifth International Conference on Differential Geometry and Its Applications, Opava, Czech Republic, Part II, 1992. P. 103-122; www.emis.de/proceedings/5ICDGA/. [6] I. S. Krasil'shchik, A. M. Vinogradov // Acta Appl. Math. 1989. V. 15. № 1-2. P. 161-209. [7] M. Marvan // Proceedings of the International Conference on Secondary Calculus and Cohomological Physics, Moscow, 1997; www.emis.de/ proceedings/SCCP97/.

Независимьй московский университет; University of Twente, the Netherlands 